\title{
Emprendedores y pymes en el Perú
}

\author{
Entrepreneurs and SMEs in Peru
}

\author{
'Víctor Gerónimo Damián López \\ Departamento Académico de Ingeniería Comercial. Universidad \\ Nacional Jorge Basadre Grohmann. Tacna - Perú. \\ E - mail: gdamianl@unjbg.edu.pe
}

\section{RESUMEN}

El presente trabajo de investigación tuvo como finalidad demostrar que las pymes, pequeñas y medianas empresas, son organizaciones económicas básicas, producto de los emprendedores y que, debido al poco o ningún apoyo del Estado, operan en la informalidad de nuestra economía. En cuanto a la metodología, el tipo de investigación es básico. El diseño de investigación es no experimental. La muestra estuvo constituida por artículos científicos. El instrumento utilizado fue la guía de análisis documental. La investigación arribó a las siguientes conclusiones: Las pymes, pequeñas y medianas empresas en el Perú, son organizaciones económicas básicas, producto de los emprendedores y que, debido al poco apoyo o ninguna intervención del Estado, operan en la informalidad. La informalidad en la economía peruana es estructural.

Palabras clave: Acceso financiero, emprendedor, Estado, formalización, informalidad, inscripción electrónica, pyme, tributación.

\begin{abstract}
The purpose of this research work was to demonstrate that SMEs, small and medium enterprises, are basic political organizations, the product of entrepreneurs and that, due to little or no State support, they operate in the informality of our economy. Regarding the methodology, the type of research is basic. The research design is non-experimental. The sample consisted of scientific articles. The instrument used was the documentary analysis guide. The investigation reached the following conclusions: The SMEs, small and medium enterprises in Peru, are basic economic organizations, product of the entrepreneur and that, due to little or no support of the State, they operate in the informality. Informality in Peruvian economy is structural.
\end{abstract}

Keywords: Financial access, entrepreneurs, State, formalization, informality, electronic registration, SMEs taxation.

\section{INTRODUCCIÓN}

Gómez (2007), en su investigación La Informalidad en la economía, algo incuestionable, concluye que la economía informal cobija gran parte de las actividades económicas en los países y merece ser estudiada debidamente, de forma que permita esclarecer con nitidez la razón del problema y así aportar a la explicación de uno de los puntos a tener en cuenta cuando de economía se habla, en términos de la racionalidad del manejo de los recursos. La economía informal debe ser asumida en una discusión seria y profunda con riqueza teórica y conceptual que brote de la economía para su más correcta interpretación.

Por otro lado, Aguirre (2016), en su investigación Factores institucionales que inciden en el proceso emprendedor de las PYMES del cantón Machala, provincia de El Oro, desde la percepción del empresario, concluye que la actitud emprendedora se relaciona con el proceso emprendedor en sus elementos de inversión

DOI: https://doi.org/10.33326/27086062.2020.1.903

Presentado: 18/03/2020, Aceptado: 19/06/2020 
inicial, acceso a recursos financieros y tecnológicos. Estos factores también muestran una interacción con los factores de ingreso al mercado, las fuentes de financiamiento, las oportunidades de negocio, la motivación del empresario, sus competencias, el acceso a recursos materiales y humanos, la experiencia laboral y la demanda del mercado como tal.

La vinculación emprendedores - pymes es inevitable en el entendido que los emprendedores son personas valientes que, al identificar una oportunidad de negocio, organizan los recursos, crean una pequeña o mediana empresa y la llevan adelante por iniciativa propia (Bojica, Ruiz, y Fuentes, 2012).

El emprendimiento es una actitud, pero también es una aptitud que permite alcanzar el propósito y la materialización de los sueños. Así, no basta la actitud de la determinación para llevar adelante un negocio, sino que se fundamente el despliegue de esfuerzos de organización y dirección, para lo cual, no todos están preparados (Bucardo, Saavedra y Camarena, 2015).

La actitud de autodeterminación y aptitud especial del emprendedor permite distinguirlo del empresario que tiene un perfil pre-establecido, un prototipo que goza de una percepción en casi todo el mundo. El emprendedor, evidentemente, tiene también un perfil y este es ser: creativo, arriesgado, flexible, corajudo; pero, lo más importantes, es autodidacta (Urbano y Toledano, 2007).

\section{CONCEPTUALIZACIÓN}

Con fines académicos, podríamos establecer diferencias entre un emprendedor y un empresario, de la siguiente manera:

\section{EMPRENDEDOR}

- Trabaja mucho

- Hace y controla el negocio

- Es bueno solucionando problemas

\section{EMPRESARIO}

- Piensa y elabora mucho

-Delega y controla resultados

-Es bueno definiendo objetivo

Soslayando estas diferencias, en el mercado tanto emprendedores como empresarios ponen en marcha todas las estrategias posibles para lograr sus objetivos. Es decir, "Todas las organizaciones necesitan una estrategia corporativa, el esquema o medio central para utilizar e integrar los recursos de producción, finanzas, investigación y desarrollo, recursos humanos y marketing para realizar la misión de la organización y lograr las metas y objetivos deseados" (Mayorga y Araujo, 1997).

El marketing se está convirtiendo en un ingrediente indispensable para el éxito empresarial. Además, influye significativamente en nuestra vida diaria. Por ejemplo, está arraigado en todo lo que hacemos, desde la ropa que vestimos hasta los anuncios televisivos que vemos; así como las páginas web por las que navegamos (Kotler y Keller, 2012).

En efecto, una vez definida la estrategia empresarial, los emprendedores o empresarios, a través de sus ejecutivos, desarrollan los programas que permitirán establecer los elementos necesarios para seguir un curso de acción.

\section{OBJETIVO GENERAL}

Demostrar que las pymes, pequeñas y medianas empresas, son organizaciones económicas básicas, producto de los emprendedores y que, debido al poco o ningún apoyo del Estado, operan en la informalidad de nuestra economía.

\section{OBJETIVOS ESPECÍFICOS}

a. Analizar la operatividad de las pymes, pequeñas y medianas empresas, en Perú.

b. Analizar cómo se presenta la informalidad en la economía peruana. 


\section{FUNDAMENTOTEÓRICO}

\section{Informalidad}

El emprendedor, agente activo del mercado, es producto de una economía y de un Estado cerrados para la iniciativa y la facilitación de los negocios. Esta es una cruda realidad de los países en vías de desarrollo como el Perú y América Latina en general, donde en promedio, más del 50 \% de su economía es informal.

Las principales causas de la informalidad son:

- Barreras burocráticas; que generan competencia desleal y baja productividad.

- Sistema tributario inadecuado; que genera evasión tributaria y baja presión tributaria.

- Rigidez laboral; que genera alto subempleo y carencia de beneficios sociales.

Estas causas ponen en agenda la urgencia de una profunda reforma del Estado. Las cifras de la informalidad dejan en la saga al Perú en América Latina y en el mundo; veamos:

- En la OCDE, el promedio es $16.3 \%$. EE. UU. se encuentra mejor ubicado a con $8.4 \%$; y peor ubicado se encuentra Grecia con $28.2 \%$.

- En Asia, el promedio es $30.4 \%$; mejor ubicado se encuentra Singapur con $13.7 \%$; y peor ubicado, Tailandia con $54.1 \%$.

- En Europa Central y del Este, el promedio es 40 \%; mejor ubicada está República Checa con $20.1 \%$; y peor ubicado, Georgia con $68 \%$.

- En América Latina, el promedio es $43 \%$; mejor ubicado Chile con $20.9 \%$; y peor ubicados, Bolivia y Perú con $68.2 \%$.

- En Perú, específicamente las pymes pertenecen al mundo informal en un 73\%; y tienen solo el $14 \%$ del empleo formal.

- El Estado peruano está convencido que los sectores de menos recursos, en ascenso, han dejado de ser el patito feo del mercado para convertirse en un mercado muy atractivo, que generan volúmenes de ventas interesantes. Ellos constituyen la fuerza que ha creado el sector informal en nuestros países. Asimismo, se ha iniciado un proceso de apoyo y, de manera reactiva, se está exonerando el pago de la formalización de las pymes con capital de una UIT, a través de los Centros de Desarrollo Empresarial de los Registros Públicos, con el concurso del Ministerio de la Producción, a través de un sistema informático SID-Emprendedor.

\section{Sistema Tributario}

Cabe señalar que, entre las causas de la informalidad también se encuentra el sistema tributario inadecuado en el Perú, como podemos mostrar en la siguiente tabla comparativa con la OCDE:

Tabla 1. Sistema tributario en el Perú

\begin{tabular}{lcc}
\hline Tipo de impuesto & OCDE(\%) & PERU(\%) \\
\hline Renta & 63 & 35.6 \\
IVA (IGV) & 34.7 & 49.1 \\
Otros & 2.3 & 15.3 \\
\hline
\end{tabular}

Como se puede apreciar, en los países desarrollados tributan más las empresas pagando el impuesto a la renta (63 $\%$ ), mientras que, en Perú, más tributan los consumidores pagando el IGV (49.1\%). El poco peso del impuesto a la renta se debe a que la mayoría de los negocios son informales. Se requiere una profunda reforma del sistema tributario peruano. Recientemente, SUNAT ha puesto en marcha un programa de apoyo a las pymes, denominado "pymes tributario", con ventajas para las empresas cuyas ventas no superen las 1700 UIT; así como el programa "IGV Justo" que permite que las empresas pueden prorrogar por 3 meses los pagos del IGV (Arellano, 2000). 


\section{RESULTADOS}

Se concluye que, en América Latina, donde se estima que existen 20 millones de emprendedores, más del $50 \%$ de la economía es informal.

La informalidad en América Latina es alta, comparada con otros continentes; y, particularmente, en el Perú es la más alta del mundo (68.3\%), lugar que comparte con Bolivia. En lo que respecta a las pymes en el Perú, se registra el $73 \%$ de informalidad. Lo cual tiene como causas principales a las barreras burocráticas, al sistema tributario inadecuado y a una rigidez laboral que, en conjunto, determinan una economía de baja productividad, baja presión tributaria y alto subempleo.

Los Estados, en una actitud reactiva, están promoviendo la formalización de los negocios y estableciendo algunas facilidades para las empresas que se han formalizado. Podemos destacar la implementación del registro electrónico de empresas en 24 horas, como en Argentina y Chile. En otros países como México, se está impulsando instituciones bancarias pro-emprendedor, buscando mayor acceso de las pymes al sistema financiero, ya que se estima que el 85 \% de las pymes se financian solos. En el Perú, "Sunat pyme" y "IGV Justo" son iniciativas alentadoras.

\section{CONCLUSIONES}

- Las pymes, pequeñas y medianas empresas, en el Perú, son organizaciones económicas básicas, producto de los emprendedores; y que debido al poco o ningún apoyo del Estado, operaran mayormente en la informalidad.

- Las pymes constituyen un soporte importante de la economía, generador de empleo y fuente de consumo interno.

- Las informalidades en la economía peruana se presentan en forma permanente y es estructural.

- El Estado actúa todavía de manera reactiva en la formalización y facilitación de las pymes.

- Según CEPAL, la data de las mipymes en América Latina es escasa y poco confiable.

\section{REFERENCIAS}

Aguirre, M. (2016). Factores institucionales que inciden en el proceso emprendedor de las PYMES del cantón Machala, provincia de El Oro, desde la percepción del empresario (tesis de posgrado). Universidad Nacional Mayor de San Marcos, Perú.

Arellano, R. (2000). Estilos de Vida en el Perú: Como somos y pensamos los peruanos del siglo XXI. Lima, Perú: Consumidores y Mercados.

Bojica, A., Ruiz, M. y Fuentes, M. (2012). La adquisición de conocimiento a través de relaciones interorganizativas y la orientación emprendedora: el papel mediador del capital social de segundo orden. Cuadernos de Economía y Dirección de la Empresa,(15), 141-153.

Bucardo, M., Saavedra, María y Camarena, M. (2015). Hacia Una Comprensión De Los Conceptos De Emprendedores Y Empresarios. Suma de Negocios, 6 (13), 98-107. Disponible en SSRN: https://ssrn.com/abstract=3019399

Gómez, L. (2007). La Informalidad en la economía, algo incuestionable. Semestre Económico, 10 (19), 47-67.

Kotler, P. y Keller, K. (2012). Dirección de Marketing. México: Pearson Educación.

Urbano, D. y Toledano, N. (2007). Invitación al emprendimiento: Una aproximación a la creación de empresas. Barcelona, Barcelona: UOC.

Mayorga, M. y Araujo, P. (1997). Marketing Estratégico en la empresa peruana. Universidad del Pacifico Centro de Investigación. 